\title{
On $G$-transitive version of perfectly meager sets
}

\author{
ANDRZEJ NOWIK
}

\begin{abstract}
We study the $G$ - invariant version of perfectly meager sets (a generalization of the notion of $\mathrm{AFC}^{\prime}$ sets). We find the necessary and sufficient conditions for the inclusion $\mathrm{AFC}_{G}^{\prime} \subseteq \mathcal{I}$. In particular, we partially characterize for which groups $G$ of automorphisms of the Cantor space every $\mathrm{AFC}_{G}^{\prime}$ set is Lebesgue null.
\end{abstract}

\section{Definitions And NOtATion}

We consider the Cantor space $2^{\omega}$ as a topological group (where $(x+$ $y)(k)=x(k)+y(k) \bmod 2)$. By $2^{<\omega}$ let us denote the collection of all finite binary sequences: $2^{<\omega}=\{f: n \rightarrow 2$ where $n \in \omega\}$

For any $s \in 2^{<\omega}$ by $[s]$ denote the base open set detemined by $s:[s]=$ $\left\{x \in 2^{\omega}: s \subseteq x\right\}$. Let Perf stand for the family of all perfect subsets of the space $2^{\omega}$. Recall that a proper collection of subsets of $2^{\omega}: \mathcal{I} \subseteq P\left(2^{\omega}\right)$ is called a $\sigma$ - ideal iff it is closed under taking subsets and countable sums. Throughout the paper we assume that every $\sigma$ - ideal $\mathcal{I}$ contains all singletons: $\forall x \in X\{x\} \in \mathcal{I}$.

Let $\mathcal{I} \subseteq P\left(2^{\omega}\right)$ be a $\sigma$ - ideal. Define the following cardinal numbers:

Definition 1. $\operatorname{cov}(\mathcal{I})=\min \left\{|\mathcal{A}|: \mathcal{A} \subseteq \mathcal{I} \wedge \bigcup \mathcal{A}=2^{\omega}\right\}$

and

$$
\operatorname{cof}(\mathcal{I})=\min \left\{|\mathcal{A}|: \mathcal{A} \subseteq \mathcal{I} \wedge \forall_{Z \in \mathcal{I}} \exists_{A \in \mathcal{A}} Z \subseteq A\right\} .
$$

Notice that we always have $\operatorname{cov}(\mathcal{I}) \leq \operatorname{cof}(\mathcal{I})$.

We assume that the reader is familiar with basic concept of arithmetic of cardinal numbers. In particular, we need the notion of cofinality; recall that an uncountable cardinal number $\kappa$ is called regular iff $\operatorname{cf}(\kappa)=\kappa$.

By $\operatorname{Hom}(X)$ we denote the group of all homeomorphisms of the topological space $X$. We always assume that $G$ is a fixed subgroup of $\operatorname{Hom}\left(2^{\omega}\right)$.

The following additional terminology will be useful in our proof.

2010 Mathematics Subject Classification. Primary: 03E15; Secondary 03E20, 28 E15.

Key words and phrases. Perfectly meager sets, strongly meager sets, AFC' sets.

Full paper. Received 17 October 2019, revised 21 January 2020, accepted 15 February 2020, available online 29 February 2020. 
For an arbitrary $g \in G$ and $Q \in P e r f$ we often abbreviate the image $g(Q)=\{g x: x \in Q\}$ and write simply $g Q$. Also for any $t \in 2^{\omega}$ and $A \subseteq 2^{\omega}$ we write $A+t=\{x+t: x \in A\}$.

We denote by $\mathcal{M}(P)$ the collection of all first category sets on $P$, where $P \in \operatorname{Perf}(X)$.

We use a letter $\mathcal{N}$ to denote the sigma ideal of Lebesgue measure zero sets of $2^{\omega}$.

We denote by $\operatorname{Trans}\left(2^{\omega}\right)$ the subgroup of all translations of $2^{\omega}$.

\section{INTRODUCTION}

Let us start with the following, classical definition:

Definition 2. A subset $S \subseteq 2^{\omega}$ is a Sierpinski set if, and only if, it is uncountable and has countable intersection with any set of measure zero.

Notice that under the assumption of Continuum Hypothesis there exists a Sierpiński set (see [9]) and, on the other hand, it is consistent that there is no Sierpiński set.

A special variation of the notion of a Sierpiński set is a $\kappa$ - Sierpiński set with respect to the $\sigma$-ideal $\mathcal{I}$, namely:

Definition 3. Suppose that $\kappa$ is a cardinal number and $\mathcal{I} \subseteq P\left(2^{\omega}\right)$ a $\sigma$ ideal. A set $X \subseteq 2^{\omega}$ is called a $\kappa$ - Sierpiński set $X$ with respect to $\mathcal{I}$ iff $|X|=\kappa$ and $\forall_{A \in \mathcal{I}}|A \cap X|<\kappa$.

Notice that if $\mathcal{T}$ is a $\sigma$-ideal (which contains singletons) and $\kappa=\operatorname{cof}(\mathcal{I})=$ $\operatorname{cov}(\mathcal{I})$ then there exists a $\kappa$ - Sierpiński set $X$ with respect to $\mathcal{I}$.

Recall the classical definition of perfectly meager sets (called also always of the first category sets):

Definition 4. A set $X$ of $2^{\omega}$ is a perfectly meager (AFC) set iff for every $P \in$ Perf, $X \cap P$ is a first category set in the topology of $P$.

The following notion of sets was first defined in [5] and then it has been studied most extensively in papers [6] and [7].

Definition 5. A set $X \subseteq 2^{\omega}$ is an $\mathrm{AFC}^{\prime}$-set if for each perfect set $P$ there exists an $\mathrm{F}_{\sigma}$-set $F$ such that $X \subseteq F$ and for each $t \in 2^{\omega},(F+t) \cap P$ is a first category set in the topology of $P$.

Notice that the notion $\mathrm{AFC}^{\prime}$ is a strengthening of the classical perfectly meager sets.

The following notion was first defined by Karel Prikry: (see [3], introduction):

Definition 6. A set $X \subseteq 2^{\omega}$ is called strongly meager (SFC) iff for every measure zero set $A \subseteq 2^{\omega}$, there exists $t \overline{\in 2^{\omega} \text {, such that }}(X+t) \cap A=\emptyset$. 
Notice that K. Prikry conjectured that the collection of strongly meager sets form a $\sigma$-ideal but it turned out that it is consistent that strongly meager sets are exactly the countable sets (see [3]) and that it is consistent that even the sum of two strongly meager sets need not be strongly meager set (see $[2])$.

It is known (see for example [5] and [7]), that $\mathrm{AFC}^{\prime} \subseteq \mathrm{AFC}$ and every strongly meager set is an $\mathrm{AFC}^{\prime}$ set.

It is also known (see [8]) that every Sierpiński set is strongly meager.

We can summarize all these inclusions in Fig. 1

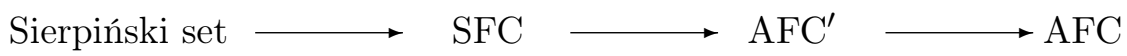

Figure 1. Basic relations.

Let us define the main notion of this article.

The $\mathrm{AFC}_{G}^{\prime}$ - sets

Suppose that $\mathrm{G}$ is a subgroup of $\operatorname{Hom}\left(2^{\omega}\right)$ and let $X$ be an arbitrary subset of $2^{\omega}$.

Definition 7. Suppose that $X \subseteq 2^{\omega}$. We write $X \in \mathrm{AFC}_{G}^{\prime}$ iff for every $Q \in P$ erf there exists $F \supseteq X, F \in \mathrm{F}_{\sigma}$ such that $\forall_{g \in G} g Q \cap F \in \mathcal{M}(g Q)$.

This notion is a natural generalization of the notion of $\mathrm{AFC}^{\prime}$ sets.

\section{Remarks:}

It is obvious that

$$
\mathrm{AFC}_{\text {Trans }\left(2^{\omega}\right)}^{\prime}=\mathrm{AFC}^{\prime}, \quad \mathrm{AFC}_{\{i d\}}^{\prime}=\mathrm{AFC}, \quad \mathrm{AFC}_{\mathrm{Hom}\left(2^{\omega}\right)}^{\prime}=\left[2^{\omega}\right]^{\leq \omega} .
$$

It is also evident that if $G_{1} \subseteq G_{2}$, then $\mathrm{AFC}_{G_{1}}^{\prime} \supseteq \mathrm{AFC}_{G_{2}}^{\prime}$.

All inclusions are summarized in Fig. 2 (where arrows denote inclusions).

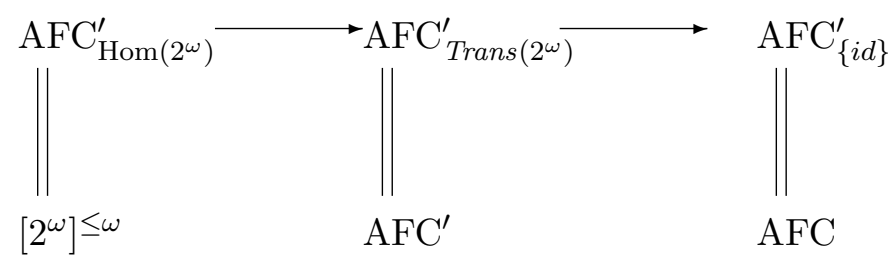

FigURE 2. Relations between various versions of perfectly meager sets.

Let us define: 
Definition 8. Suppose that $\mathcal{I}$ is a $\sigma$ - ideal of subsets of the space $2^{\omega}$.

We say that a group $G \leq \operatorname{Hom}\left(2^{\omega}\right)$ has the $(E m)_{\mathcal{I}}$ property iff there exists a perfect set $Q \in \operatorname{Perf}$ such that for each $P \in \operatorname{Perf} \backslash \mathcal{I}$ there exists $g \in G$ such that $P \cap g Q \notin \mathcal{M}(g Q)$.

\section{Remarks:}

One can prove that $\operatorname{Trans}\left(2^{\omega}\right)$ does not have the $(E m)_{\mathcal{N}}$ property.

Without loss of generality we may assume that in Definition $8 P$ is only closed set such that $P \notin \mathcal{I}$.

We will start with the following theorem.

Theorem 1. Let $\mathcal{I}$ be an arbitrary $\sigma$ - ideal of subsets of $2^{\omega}$ such that $\forall x \in 2^{\omega}\{x\} \in \mathcal{I}$.

Moreover, let $G \leq \operatorname{Hom}\left(2^{\omega}\right)$ be a subgroup of $\operatorname{Hom}\left(2^{\omega}\right)$ with the property $(E m)_{\mathcal{I}}$.

Then we have: $\mathrm{AFC}_{G}^{\prime} \subseteq \mathcal{I}$.

Proof. Let $X \subseteq 2^{\omega}$ be a set such that $X \notin \mathcal{I}$. By the definition of the notion $(E m)_{\mathcal{I}}$ there is a perfect set $Q$ such that for each closed $E \notin \mathcal{I}$ we have $\exists_{g \in G} E \cap g Q \notin \mathcal{M}(g Q)$.

Let $F \subseteq 2^{\omega}$ be an $F_{\sigma}$ set such that $X \subseteq F$. We have

$$
F=\bigcup_{n<\omega} F_{n},
$$

where $\operatorname{cl}\left(F_{n}\right)=F_{n}$, so there exists $n_{0}<\omega$ such that $F_{n_{0}} \notin \mathcal{I}$. Now there exists $g \in G$ such that $F_{n_{0}} \cap g Q$ is not meager in $g Q$. So we conclude, that $X$ is not an $\mathrm{AFC}_{G}^{\prime}$ set.

The implication given in Theorem 1 is reversible under some additional set theoretical assumptions. Indeed, we have the following theorem.

Theorem 2. Let us assume like in Theorem 1 that $\mathcal{I}$ is an arbitrary $\sigma$-ideal of subsets of $2^{\omega}$ such that $\forall_{x \in 2^{\omega}}\{x\} \in \mathcal{I}$ and $G \leq \operatorname{Hom}\left(2^{\omega}\right)$ is a subgroup of $\operatorname{Hom}\left(2^{\omega}\right)$. Moreover, assume that

(1) $\operatorname{cof}(\mathcal{I})=\operatorname{cov}(\mathcal{I}) \leq \operatorname{non}\left(\mathrm{AFC}_{G}^{\prime}\right)$,

(2) $\forall_{P \in P e r f} \backslash \mathcal{I}^{\exists}|C| \leq \omega 2^{\omega} \backslash(P+C) \in \mathcal{I}$,

(3) $\operatorname{Trans}\left(2^{\omega}\right) \subseteq G$.

Then the following conditions are equivalent:

(1) $\mathrm{AFC}_{G}^{\prime} \subseteq \mathcal{I}$

(2) $G$ fulfills $(E m)_{\mathcal{I}}$.

Proof. Theorem 1 gives us immediately the implication $(2) \Rightarrow(1)$.

Now suppose that $G$ fulfills $\neg(E m)_{\mathcal{I}}$. Since $\kappa=\operatorname{cof}(\mathcal{I})=\operatorname{cov}(\mathcal{I})$ and $\mathcal{I}$ contains singletons we conclude that there exists a $\kappa$-Sierpiński set $X$ with respect to $\mathcal{I}$ (see Def. 3). Let $Q \in \operatorname{Perf}$ be arbitrary. From the assumption 
$\neg(E m)_{\mathcal{I}}$ there exists a perfect set $P$ such that $P \notin \mathcal{I}$ and $\forall_{g \in G} g Q \cap P \in$ $\mathcal{M}(g Q)$. Pick a countable set $C \subseteq 2^{\omega}$ such that $2^{\omega} \backslash(C+P) \in \mathcal{I}$.

We have

$$
X=\left[\left[2^{\omega} \backslash(P+C)\right] \cap X\right] \cup[(P+C) \cap X] .
$$

Since $2^{\omega} \backslash(P+C) \in \mathcal{I}$ we obtain $\left|\left[2^{\omega} \backslash(P+C)\right] \cap X\right|<\kappa$. Moreover, if $c \in C$ and $g \in G$, then $h Q \cap P \in \mathcal{M}(h Q)$, where $h \in G$ is defined by $h(x)=g(x)-c$. Hence $g Q \cap(P+c) \in \mathcal{M}(g Q)$, thus $g Q \cap(P+C) \in \mathcal{M}(g Q)$ for each $g \in G$.

Since $\kappa \leq \operatorname{non}\left(\mathrm{AFC}_{G}^{\prime}\right)$ we obtain $\left[2^{\omega} \backslash(P+C)\right] \cap X \in \mathrm{AFC}_{G}^{\prime}$, so there exists $E \in \mathrm{F}_{\sigma}, E \supseteq X \backslash(P+C)$ such that $\forall_{g \in G} g Q \cap E \in \mathcal{M}(g Q)$. Finally, define $E^{*}=E \cup(P+C)$. It is easy to see that $X \subseteq E^{*}$ and $\forall_{g \in G} g Q \cap E^{*} \in \mathcal{M}(g Q)$. Hence $X \in \mathrm{AFC}_{G}^{\prime}$ and the proof is completed, since $\mathrm{X}$ does not belong to $\mathcal{I}$.

Unfortunately, we don't know whether this theorem is true under weaker assumptions. Thus we think that the following question may be of some interest.

Question 3. Can we prove the equivalence from Theorem 2 under weaker assumptions?

For any $\mathcal{F} \subseteq$ Perf let us define the following cardinal coefficient:

Definition 9. $\operatorname{Em}(\mathcal{F}, G)=\min \left\{|\mathcal{G}|: \mathcal{G} \subseteq \operatorname{Perf} \wedge \forall_{P \in \mathcal{F}} \exists g \in G \exists_{Q \in \mathcal{G}} g Q \subseteq P\right\}$

Let us formulate a characterization of the property $(E m)$ in terms of the coefficient $\operatorname{Em}(\mathcal{F}, G)$.

Assume that $G$ has the property that for each $x \in 2^{\omega}$ the orbit $G x$ is dense in $2^{\omega}$. Then the following conditions are equivalent:

(1) $G$ fulfills $(E m)_{\mathcal{I}}$;

(2) $|E m(\operatorname{Perf} \backslash \mathcal{I}, G)| \leq \aleph_{0}$.

We will need the following technical lemma (folklore for the group $G=$ $\left.\operatorname{Trans}\left(2^{\omega}\right)\right)$ :

Lemma 1. If $G \leq \operatorname{Hom}\left(2^{\omega}\right)$ is a group such that for each $x \in 2^{\omega}, G x$ is dense in $2^{\omega}$, then for every sequence $\left\langle Q_{n}\right\rangle$ of perfect subsets of $2^{\omega}$ there exists a perfect $P \in$ Perf such that $\forall_{n \in \omega} \exists_{g \in G} g Q_{n} \cap P \notin \mathcal{M}(P)$.

Proof. Let $v_{k}=[(0, \ldots, 0,1)](0 k$ times $)$. For each $k$ choose $x_{k} \in Q_{k}$ and $g_{k} \in G$ such that $g_{k} x_{k} \in V_{k}$. Define $P=\overline{\bigcup_{k \in \omega} g_{k} Q_{k} \cap V_{k}}$, then $P$ is a perfect set and if $k \in \omega$ then $g_{k} Q_{k} \cap P \supseteq g_{k} Q_{k} \cap V_{k} \notin \mathcal{M}(P)$.

Proof. (1) $\rightarrow(2)$

Assume that $G$ has the $(E m)_{\mathcal{I}}$ property, i.e. there exists $Q \in$ Perf such that $\forall_{P \in \operatorname{Perf} \backslash \mathcal{I}} \exists_{g \in G} P \cap g Q \notin \mathcal{M}(g Q)$. Let us define perfect sets: $\mathcal{G}=\{Q \cap$ $\left.[s]: Q \cap[s] \neq \emptyset \wedge s \in 2^{<\omega}\right\}$. Then $|\mathcal{G}| \leq \aleph_{0}$ and if $P \in \operatorname{Perf} \backslash \mathcal{I}$ then there exists $g \in G$ such that $P \cap g Q \notin \mathcal{M}(g Q)$, so $P \cap g Q \supseteq W \cap g Q \neq \emptyset$ for 
some open set $W$. Then $g^{-1}[W] \cap Q \not \emptyset$ so there exists $Q_{1} \in \mathcal{G}$ such that $Q_{1} \subseteq g^{-1}[W] \cap Q$. Hence $g\left[Q_{1}\right] \subseteq W \cap g[Q] \subseteq P \cap g[Q]$. This proves (2).

$(2) \rightarrow(1)$.

Next we give an useful characterization of the property $(E m)_{\mathcal{N}}$.

Theorem 4. Let $G$ be a subgroup of $\operatorname{Hom}\left(2^{\omega}\right)$ which contains the subgroup $\operatorname{Trans}\left(2^{\omega}\right)$. The following two conditions are equivalent:

(1) $\neg(E m)_{\mathcal{N}}$,

(2) For every $Q \in$ Perf and for every $\epsilon>0$ there exists an open set $U$, such that $\mu(U)<\epsilon$ and $\forall_{g \in G} g Q \cap U \neq \emptyset$

Proof. (1) $\Rightarrow(2)$

Assume that $\forall_{Q \in P e r f} \exists_{P \in P e r f} \forall_{g \in G} g Q \cap P \in \mathcal{M}(g Q)$

Let $Q \in P$ Perf be any perfect set and let $\epsilon>0$. Pick a perfect set $P$, $\mu(P)>0$ such that $\forall_{g \in G} g Q \cap P \in \mathcal{M}(g Q)$. We can find finite $C \subseteq 2^{\omega}$ such that $\mu\left(2^{\omega} \backslash(C+P)\right)<\epsilon$. Now put $U=2^{\omega} \backslash(C+P)$.

By way of contradiction suppose that there exists $g \in G$ such that $g Q \cap$ $U=\emptyset$. Then $g Q \subseteq C+P$, hence there exists $c_{0} \in C$ and an open set $I$ such that $\emptyset \neq I \cap g Q \subseteq P+c_{0}$. Define $h(x)=g(x)-c_{0}$, obviously $h \in G$. Next, $h Q=g Q-c_{0}$ thus $\emptyset \neq h Q \cap\left(I-c_{0}\right) \subseteq P$, which is a contradiction with $h Q \cap P \in \mathcal{M}(h Q)$.

$(2) \Rightarrow(1)$

Assume (2). Let $R$ be any perfect set. Let $\left\{I_{m}\right\}_{m<\omega}$ be an enumeration of all basic clopen sets of $2^{\omega}$. Let

$$
\epsilon_{m}=\frac{1}{2^{m+2}} .
$$

For any $m<\omega$ we choose, using the assumption (2), an open set $U_{m}$ such that

$$
\forall_{g \in G} R \cap I_{m} \neq \emptyset \Rightarrow U_{m} \cap g\left(R \cap I_{m}\right) \neq \emptyset
$$

and $\mu\left(U_{m}\right)<\epsilon_{m}$. This can be done, since $I_{m} \cap R$ is a perfect or an empty set.

Now put

We see that

$$
U=\bigcup_{m<\omega} U_{m}
$$

$$
\mu(U) \leq \sum_{m<\omega} \frac{1}{2^{m+2}} \leq 2 \cdot \frac{1}{4}<1 .
$$

Define $F=2^{\omega} \backslash U$, then we have $\mu(F)>0$ so choose a perfect $P \subseteq F$ of positive measure.

Let $g \in G$ and $I_{m_{0}}$ be given such that $R \cap I_{m_{0}} \neq \emptyset$. 
Now $U_{m_{0}} \cap g\left(R \cap I_{m_{0}}\right) \neq \emptyset$. Moreover, since $U_{m_{0}} \cap P=\emptyset$ we obtain that $g\left(R \cap I_{m_{0}}\right) \nsubseteq P$. This means that (1) holds.

Notice that in the proof of implication $(2) \Rightarrow(1)$ we did not use the assumption that $\operatorname{Trans}\left(2^{\omega}\right) \leq G$.

In the next part we will prove theorems about relations between $\mathrm{AFC}_{G}^{\prime}$ and different classes of peculiar small sets of the real line.

Theorem 5. Assume that $G$ is a subgroup of $\operatorname{Hom}\left(2^{\omega}\right)$ which contains $\operatorname{Trans}\left(2^{\omega}\right)$. If $G$ fulfills $\neg(E m)_{\mathcal{N}}$, then every strongly meager set is an $\mathrm{AFC}_{G}^{\prime}$ set.

Proof. Let X be a strongly meager set and let $Q$ be an arbitrary perfect set. Since $\neg(E m)_{\mathcal{N}}$ we obtain that there exists a perfect set $P$ such that $\mu(P)>0$ and $\forall_{g \in G} g(Q) \cap P \in \mathcal{M}(g(Q))$. Let $C \subseteq 2^{\omega}$ be a countable set such that $2^{\omega} \backslash(P+C) \in \mathcal{N}$. Then there exists $x_{0}$ such that $\left(x_{0}+X\right) \cap\left[2^{\omega} \backslash(P+C)\right]=$ $\emptyset$, so $x_{0}+X \subseteq P+C$, hence $X \subseteq P+C-x_{0}$. Let $g \in G$ be an arbitrary and let $c \in C$. Define $h \in G$ by $h(x)=g(x)-c+x_{0}$. Then $h(Q) \cap P \in \mathcal{M}(h(Q))$, hence $\left(g(Q)-c+x_{0}\right) \cap P \in \mathcal{M}\left(g(Q)-c+x_{0}\right)$, thus $g(Q) \cap\left(P+c-x_{0}\right) \in \mathcal{M}(g(Q))$. Since $c \in C$ was taken arbitrary, we conclude that $g(Q) \cap\left(P+C-x_{0}\right) \in \mathcal{M}(g(Q))$. This implies that $X \in \mathrm{AFC}_{G}^{\prime}$, since $P+C-x_{0} \in \mathrm{F}_{\sigma}$.

\section{Remark:}

This implication is reversible under $\mathrm{CH}$. Namely:

Theorem 6. Suppose that $G \leq \operatorname{Hom}\left(2^{\omega}\right)$ and assume that $G$ has the $(E m)_{\mathcal{N}}$ property. Moreover, assume $\mathrm{CH}$. Then there exists a strongly meager set $X \subseteq 2^{\omega}$ such that $X \notin \mathrm{AFC}_{G}^{\prime}$.

Proof. Let $X \subseteq 2^{\omega}$ be arbitrary Sierpiński set. Then $X$ is strongly meager $([8])$. From the $(E m)_{\mathcal{N}}$ property we obtain that there exists $Q \in \operatorname{Perf}$ such that

$$
\forall_{P \in P \operatorname{erf}} \backslash \mathcal{N}^{\exists} \exists_{g \in G} P \cap g(Q) \notin \mathcal{M}(g Q) .
$$

Suppose that $E$ is an $\mathrm{F}_{\sigma}$-set such that $X \subseteq E$. Since $X \notin \mathcal{N}$ it follows that $E \notin \mathcal{N}$. Hence there exists $P \in \operatorname{Perf} \backslash \mathcal{N}$ such that $P \subseteq E$

Therefore $\exists_{g \in G} P \cap g(Q) \notin \mathcal{M}(g Q)$, hence $E \cap g(Q) \notin \mathcal{M}(g(Q))$. This yields $X \notin \mathrm{AFC}_{G}^{\prime}$, which finishes the proof.

Corollary 1. Assume that $\operatorname{cov}(\mathcal{N})=\operatorname{cof}(\mathcal{N})$ and $\operatorname{cov}(\mathcal{N})$ is a regular cardinal. Let $G \leq \operatorname{Hom}\left(2^{\omega}\right)$ and suppose that $\operatorname{Trans}\left(2^{\omega}\right) \leq G$. Then the following conditions are equivalent:

(1) $G$ has the $(E m)_{\mathcal{N}}$ property.

(2) $\mathrm{AFC}_{G}^{\prime} \subseteq \mathcal{N}$. 
Proof. The implication $(1) \Rightarrow(2)$ follows immediately from Theorem 1 . Assume $\neg(E m)_{\mathcal{N}}$. Since $\operatorname{cov}(\mathcal{N})=\operatorname{cof}(\mathcal{N})$, there exists a $\operatorname{cof}(\mathcal{N})-$ Sierpiński set. By Lemma 8.5.4 from [1] if there exists a $\kappa$-Sierpiński set and $\operatorname{cf}(\kappa)=\kappa>\omega$, then every set of size $<\kappa$ is strongly meager. Hence by Theorem 5 we conclude that $\operatorname{non}\left(\mathrm{AFC}_{G}^{\prime}\right) \geq \operatorname{cof}(\mathcal{N})$ thus all assumptions of Theorem 2 are satisfied.

\title{
REFERENCES
}

[1] T. Bartoszyński, H. Judah, Set Theory: on the strucure of the real line, A. K. Peters, Wellesley, Mass., 1995.

[2] T. Bartoszyński, S. Shelah, Strongly meager sets do not form an ideal, Journal of Mathematical Logic, 1 (1) (2001), 1-34.

[3] T.J. Carlson, Strong measure zero and strongly meager sets, Proceedings of The American Mathematical Society, 118 (2) (1993), 577-586.

[4] A. Nowik, Remarks about transitive version of perfectly meager sets, Real Analysis Exchange, 22 (1) (1996/97), 406-412.

[5] A. Nowik, M. Scheepers, T. Weiss, The algebraic sum of sets of real numbers with strong measure zero sets, Journal of Symbolic Logic, 63 (1) (1998), 301-324.

[6] A. Nowik, T. Weiss, Not every Q-set is perfectly meager in the transitive sense, Proceedings of The American Mathematical Society, 128 (10) (2000), 3017-3024.

[7] A. Nowik, T. Weiss, The algebraic sum of a set of strong measure zero and a perfectly meager set revisited, East-West Journal of Mathematics, 2 (2) (2000), 191-194.

[8] J. Pawlikowski, All Sierpiński sets are strongly meager, Archive for Mathematical Logic, 35 (1996) 281-285.

[9] W. Sierpiński, Sur l'hypothèse du continu $\left(2^{\aleph_{0}}=\aleph_{1}\right)$, Fundamenta Mathematicae, 5 (1) (1924), 177-187.

\author{
ANDrzeJ NowiK \\ UNIVERSITY OF GDAŃSK \\ InSTITUTE OF MATHEMATICS \\ Wita Stwosza 57 \\ $80-952$ GDAŃSK \\ PolAnd \\ E-mail address: andrzej.nowik@ug.gda.pl
}

